Georgian Mathematical Journal

Volume 12 (2005), Number 2, 273-282

\title{
SEMI-SLANT SUBMANIFOLDS OF A LOCALLY PRODUCT MANIFOLD
}

\author{
HONGXIA LI AND XIMIN LIU
}

\begin{abstract}
In the present paper, we define and study the slant, bi-slant and semi-slant submanifolds of a locally product manifold. We give some characterization theorems for slant submanifolds and semi-slant submanifolds. Moreover, we obtain integrability conditions for the distributions which are involved in the definition of semi-slant submanifolds. We also get some results about mixed totally geodesic submanifolds.
\end{abstract}

2000 Mathematics Subject Classification: 53C40, 53C25, 53C15.

Key words and phrases: Locally product manifold, slant submanifold, semi-slant submanifold, bi-slant submanifold.

\section{INTRODUCTION}

Let $(\widetilde{M}, g, F)$ be a $C^{\infty}$ differentiable almost product Riemannian manifold, where $g$ is a Riemannian metric and $F$ is a non-trivial tensor field of type $(1,1)$. Moreover, $g$ and $F$ satisfy the following conditions:

$$
F^{2}=I,(F \neq \pm I), \quad g(F X, F Y)=g(X, Y), \quad X, Y \in T \widetilde{M},
$$

where I is the identity map and $T \widetilde{M}$ is the Lie algebra of vector fields on $\widetilde{M}$.

We denote by $\widetilde{\nabla}$ the Levi-Civita connection on $\widetilde{M}$ with respect to $g$ and furthermore we assume that $\widetilde{M}$ is a locally product manifold, that is

$$
\widetilde{\nabla} F=0, \quad X \in T \widetilde{M} .
$$

Locally product manifolds are a class of important manifolds introduced by S. Tachibana [1] in the early 60s. After that, many authors discussed this class of manifolds. Adati [2] defined and studied invariant, anti-invariant and noninvariant submanifolds of locally product manifolds, while Bejancu [3] studied semi-invariant submanifolds which correspond to CR-submanifolds of a Kaehlerian manifold [4]. Recently, Liu and Shao [5] have defined and studied skew semi-invariant submanifolds and many related interesting results have been obtained.

Since B. Y. Chen introduced the theory of slant immersions in complex geometry (see [6]), the differential geometry of slant submanifolds has shown an increasing development. Recently, N. Papaghiuc has introduced in [7] a class of submanifolds in an almost Hermitian manifold, called semi-slant submanifolds.

The purpose of this paper is to define and study three new classes of submanifolds of a locally product manifold, i.e., slant submanifolds, bi-slant submanifolds and semi-slant submanifolds. We will focus our attention mainly on 
semi-slant submanifolds which contain semi-invariant submanifolds as a special case.

In Section 2, we review the basic formulas for locally product manifolds and submanifolds in locally product manifolds. Slant immersions are introduced in Section 3. In Section 4, we define slant distributions and introduce a more general class of submanifolds, that is bi-slant submanifolds. We also give a sufficient and necessary condition for a distribution to be slant. In Section 5, we define semi-slant immersions and obtain a useful characterization of semislant submanifolds in locally product manifolds.

\section{Preliminaries}

Let $M$ be a Riemannian manifold isometrically immersed in $\widetilde{M}$ and denote by the same symbol $g$ the Riemannian metric induced on $M$. Let $T M$ be the Lie algebra of vector fields in $M$ and $T^{\perp} M$ the set of all vector fields normal to $M$. Denote by $\nabla$ the Levi-Civita connection of $M$. Then the Gauss-Weigarten formulas are given by

$$
\begin{gathered}
\widetilde{\nabla}_{X} Y=\nabla_{X} Y+\sigma(X, Y), \\
\widetilde{\nabla}_{X} V=-A_{V} X+\nabla_{X}^{\perp} V
\end{gathered}
$$

for any $X, Y \in T M$ and any $V \in T^{\perp} M$, where $\nabla^{\perp}$ is the connection in the normal bundle, $\sigma$ is the second fundamental form of $M$, and $A_{V}$ is the Weingarten endomorphism associated with $V$. The second fundamental form $\sigma$ and the shape operator $A$ are related by

$$
g\left(A_{V} X, Y\right)=g(\sigma(X, Y), V)
$$

For any $X \in T M$, we write

$$
F X=T X+C X
$$

where $T X$ is the tangential component of $F X$ and $C X$ is the normal component of $F X$.

Similarly, for any $V \in T^{\perp} M$, we have

$$
F V=t V+n V
$$

where $t V$ (resp. $n V$ ) is the tangential component (resp. normal component) of $F V$. From $F\left(\widetilde{\nabla}_{X} Y\right)=\widetilde{\nabla}_{X} F Y$ and $(2.1),(2.2),(2.5)$ we have

$$
\begin{aligned}
& T \nabla_{X} Y+C \nabla_{X} Y+t \sigma(X, Y)+n \sigma(X, Y) \\
& =\nabla_{X} T Y+\sigma(X, T Y)-A_{C Y} X+\nabla_{X}^{\perp} C Y,
\end{aligned}
$$

for $X, Y \in T M$. Comparing the tangential and normal components in (2.6) we obtain

$$
\begin{aligned}
& T \nabla_{X} Y=\nabla_{X} T Y-t \sigma(X, Y)-A_{C Y} X \\
& C \nabla_{X} Y=\nabla_{X}^{\perp} C Y-n \sigma(X, Y)+\sigma(X, T Y),
\end{aligned}
$$

for $X, Y \in T M$. 
We define the covariant derivatives of $T$ and $C$ as follows

$$
\begin{aligned}
& \left(\nabla_{X} T\right) Y=\nabla_{X} T Y-T \nabla_{X} Y, \\
& \left(\nabla_{X} C\right) Y=\nabla_{X}^{\perp} C Y-C \nabla_{X} Y,
\end{aligned}
$$

for all $X, Y \in T M$.

Using (2.7) we have

$$
\begin{aligned}
& \left(\nabla_{X} T\right) Y=t \sigma(X, Y)+A_{C Y} X \\
& \left(\nabla_{X} C\right) Y=n \sigma(X, Y)-\sigma(X, T Y) .
\end{aligned}
$$

Let $D_{1}$ and $D_{2}$ be two distributions defined on a manifold $M$. We say that $D_{1}$ is parallel to $D_{2}$, if for all $X \in D_{2}$ and $Y \in D_{1}$ we have $\nabla_{X} Y \in D_{1}$. $D_{1}$ is called parallel if for $X \in T M$ and $Y \in D_{1}$, we have $\nabla_{X} Y \in D_{1}$. It is easy to verify that $D_{1}$ is parallel if and only if the orthogonal complementary distribution of $D_{1}$ is also parallel.

Let $M$ be a submanifold of $\widetilde{M}$. A distribution $D$ on $M$ is said to be totally geodesic if for all $X, Y \in D$ we have $\sigma(X, Y)=0$. In this case we also say that $M$ is $D$-totally geodesic. For two distributions $D_{1}$ and $D_{2}$ defined on $M$, we say that $M$ is $D_{1}-D_{2}$ mixed totally geodesic if for all $X \in D_{1}$ and $Y \in D_{2}$ we have $\sigma(X, Y)=0$.

The submanifold $M$ is said to be invariant if $C$ is identically zero, that is, $F X \in T M$ for any $X \in T M$. On the other hand, $M$ is said to be an antiinvariant submanifold if $T$ is identically zero, that is, $F X \in T^{\perp} M$ for any $X \in$ $T M . M$ is called a semi-invariant submanifold if there exists two orthogonal distributions $D_{1}$ and $D_{2}$ on $M$, such that:

(a) $T M=D_{1} \oplus D_{2}$,

(b) the distribution $D_{1}$ is invariant, i.e., $F D_{1}=D_{1}$,

(c) the distribution $D_{2}$ is anti-invariant, i.e., $F D_{2}=T^{\perp} M$.

\section{Slant Immersions}

Let $M$ be a Riemannian manifold, isometrically immersed in a locally product manifold $(\widetilde{M}, g, F)$. For each nonzero vector $X$ tangent to $M$ at $x$, we denote by $\theta(X)$ the angle between $F X$ and $T_{x} M$.

Definition 3.1. $M$ is said to be slant if the angle $\theta(X)$ is constant, which is independent of the choice of $x \in M$ and $X \in T M$. The angle $\theta$ of a slant immersion is called the slant angle of the immersion.

Invariant and anti-invariant immersions are slant immersions with slant angles $\theta=0$ and $\theta=\pi / 2$, respectively.

The following theorems are useful characterization of slant submanifolds in a locally product manifold.

Theorem 3.1. Let $M$ be a submanifold of a locally product manifold $\widetilde{M}$. Then $M$ is slant if and only if there exists a constant $\lambda \in[0,1]$ such that $T^{2}=\lambda I$. Furthermore, in this case, if $\theta$ is the slant angle of $M$, it satisfies $\lambda=\cos ^{2} \theta$. 
Proof. Suppose that $M$ is a slant submanifold. Then for any $X \in T M$ we have

$$
g\left(T^{2} X, X\right)=g(T X, T X)=\cos ^{2} \theta g(F X, F X)=g\left(\cos ^{2} \theta X, X\right),
$$

by using $\frac{|T X|}{|F X|}=\cos \theta$, where $\theta$ is the slant angle. Furthermore, for any $Y \in T M$, we have,

$$
\begin{array}{r}
g\left(\cos ^{2} \theta(X+Y), X+Y\right)=g\left(T^{2}(X+Y), X+Y\right) \\
=g\left(T^{2} X, X\right)+g\left(T^{2} Y, Y\right)+2 g\left(T^{2} X, Y\right) .
\end{array}
$$

On the other hand,

$$
g\left(\cos ^{2} \theta(X+Y), X+Y\right)=g\left(\cos ^{2} \theta X, X\right)+g\left(\cos ^{2} \theta Y, Y\right)+2 g\left(\cos ^{2} \theta X, Y\right),
$$

By comparing (3.1) and (3.2) we have

$$
g\left(T^{2} X, Y\right)=g\left(\cos ^{2} \theta X, Y\right)
$$

for any $X, Y \in T M$. Let $\lambda=\cos ^{2} \theta$, then $\lambda \in[0,1]$ and $T^{2}=\lambda I$.

Conversely, suppose that there exists a constant $\lambda \in[0,1]$ such that $T^{2}=\lambda I$. Then for any $X \in T M$, we have

$$
\cos \theta(X)=\frac{g(F X, T X)}{|F X||T X|}=\frac{g\left(X, T^{2} X\right)}{|F X||T X|}=\lambda \frac{g\left(X, F^{2} X\right)}{|F X||T X|} .
$$

On the other hand, since $\cos \theta(X)=\frac{|T X|}{|F X|}$ and by using (3.3), we obtain $\cos ^{2} \theta(X)=\lambda$. Hence $\theta(X)$ is a constant and so $M$ is slant.

Corollary 3.1. Let $M$ be a slant submanifold of a locally product manifold $\widetilde{M}$ with slant angle $\theta$. Then for any $X, Y \in T M$ we have

$$
g(T X, T Y)=\cos ^{2} \theta g(X, Y), \quad g(C X, C Y)=\sin ^{2} \theta g(X, Y) .
$$

Since for any submanifold of a locally product manifold we have $t C+T^{2}=I$, (i.e., $C T+n C=0$ ), by using (1.1), (2.4), (2.5), we can obtain the following result.

Corollary 3.2. Let $M$ be a submanifold of a locally product manifold $\widetilde{M}$. Then $M$ is slant if and only if there exists a constant $\lambda \in[0,1]$ such that $t C=\lambda I$. Furthermore, if $\theta$ is the slant angle of $M$, it satisfies $\lambda=\sin ^{2} \theta$.

\section{Slant Distributions and Bi-Slant Submanifolds}

From now on, let $M$ be a Riemannian manifold, isometrically immersed in locally product manifold $(\widetilde{M}, g, F)$.

Definition 4.1. We call a differentiable distribution $\nu$ on $M$ a slant distribution if for each $x \in M$ and each nonzero vector $X \in \nu_{x}$, the angle $\theta_{\nu}$ between $F X$ and $\nu_{x}$ is a constant which is independent of the choice of $x \in M$ and $X \in \nu_{x}$. In this case, the constant angle $\theta_{\nu}$ is called the slant angle of the distribution $\nu$.

Next, we will give a sufficient and necessary condition for a distribution to be slant. 
Theorem 4.1. Let $\nu$ be a distribution on $M$. Then $\nu$ is slant if and only if there exists a constant $\lambda \in[0,1]$ such that $\left(P_{1} T\right)^{2} X=\lambda X$ for any $X \in \nu$, where $P_{1}$ denotes the orthogonal projection on $\nu$. Furthermore in this case, $\lambda=\cos ^{2} \theta_{\nu}$.

Proof. Suppose that there exists a constant $\lambda \in[0,1]$ such that $\left(P_{1} T\right)^{2} X=\lambda X$ for any $X \in \nu$, and $P_{2}$ is the orthogonal projection on $\nu^{\perp}$. For any $X \in \nu$, we have

$$
\begin{aligned}
F X & =T X+C X=P_{1} T X+P_{2} T X+C X \\
\cos \theta_{\nu} & =\frac{g\left(F X, P_{1} T X\right)}{|F X|\left|P_{1} T X\right|}=\frac{g\left(X, F P_{1} T X\right)}{|F X|\left|P_{1} T X\right|}=\frac{g\left(X,\left(P_{1} T\right)^{2} X\right)}{|F X|\left|P_{1} T X\right|} \\
& =\lambda \frac{g\left(X, F^{2} X\right)}{|F X|\left|P_{1} T X\right|}=\lambda \frac{|F X|}{\left|P_{1} T X\right|} .
\end{aligned}
$$

On the other hand, $\cos \theta_{\nu}=\frac{\left|P_{1} T X\right|}{|F X|}$; then $\cos ^{2} \theta_{\nu}=\lambda, \theta_{\nu}$ is a constant, i.e., $\nu$ is slant. Conversely, since $\left|P_{1} T X\right|=\cos \theta_{\nu}|F X|$, we have

$$
g\left(X,\left(P_{1} T\right)^{2} X\right)=\cos \theta_{\nu}|F X|\left|P_{1} T X\right|=\cos ^{2} \theta_{\nu}|F X|^{2}=g\left(X, \cos ^{2} \theta_{\nu} X\right),
$$

which means $\left(P_{1} T\right)^{2} X=\cos ^{2} \theta_{\nu} X$.

Definition 4.2. We say $M$ is a bi-slant submanifold of $\widetilde{M}$ if there exist two orthogonal distributions $D_{1}$ and $D_{2}$ on $M$ such that:

(a) $T M$ admits the orthogonal direct decomposition $T M=D_{1} \oplus D_{2}$.

(b) For any $i=1,2, D_{i}$ is a slant distribution with slant angle $\theta_{i}$.

Given a bi-slant submanifold $M$, we can write, for any $X \in T M$,

$$
X=P_{1} X+P_{2} X
$$

where $P_{i}$ denotes the component of $X$ in $D_{i}$ for any $i=1,2$. In particular, if $X \in D_{i}$, then we obtain $X_{i}=P_{i} X$. If we define $T_{i}=P_{i} \circ T$, then we have

$$
F X=T_{1} X+T_{2} X+C X
$$

for any $X \in T M$.

Proposition 4.1. Let $M$ be a bi-slant submanifold with angles $\theta_{1}=\theta_{2}=\theta$. If $g(F X, Y)=0$, for any $X \in D_{1}, Y \in D_{2}$, then $M$ is slant with angle $\theta$.

Proof. For all $X \in D_{1}, Y \in D_{2}$, since $g(F X, Y)=0$, we have

$$
g(T X, Y)=g(F X, Y)=0
$$

then $T X \in D_{1}$. Similarly, we can obtain $T Y \in D_{2}$. For any $X \in T M=$ $D_{1} \oplus D_{2}$, there must be $X_{1} \in D_{1}, X_{2} \in D_{2}$ such that $X=X_{1}+X_{2}$, and $\cos ^{2} \theta_{1}=\frac{\left|T X_{1}\right|^{2}}{\left|F X_{1}\right|^{2}}, \cos ^{2} \theta_{2}=\frac{\left|T X_{2}\right|^{2}}{\left|F X_{2}\right|^{2}}$. Since $\theta_{1}=\theta_{2}=\theta$, we have

$$
\frac{g(T X, T X)}{g(F X, F X)}=\frac{g\left(T X_{1}, T X_{1}\right)+g\left(T X_{2}, T X_{2}\right)}{g\left(F X_{1}, F X_{1}\right)+g\left(F X_{2}, F X_{2}\right)} .
$$

Hence $M$ is slant with angle $\theta$. 
Lemma 4.1. Suppose that there exist two orthogonal distributions $D_{1}$ and $D_{2}$ on $M$, such that $T M=D_{1} \oplus D_{2}$. Then $D_{1}$ is invariant if and only if it is slant with angle $\theta_{1}=0$. Moreover, in this case, $T X=T_{2} X$ for any $X \in D_{2}$.

Proof. It is clear that if $D_{1}$ is invariant, then it is slant with zero angle. The converse is easy to prove. Since $\cos \theta=1=\frac{\left|T_{1} X\right|}{|F X|}$, we have

$$
\left|T_{1} X\right|=|F X|=\sqrt{\left|T_{1} X\right|^{2}+\left|T_{2} X\right|^{2}+|C X|^{2}},
$$

thus $\left|T_{2} X\right|=|C X|=0$ for any $X \in T M$. Consequently, $F X=T_{1} X \in D_{1}$ and we know that $D_{1}$ is invariant. On the other hand, if $D_{1}$ is invariant, then we have

$$
g(T X, Y)=g(X, F Y)=0
$$

for any $X \in D_{2}$ and $Y \in D_{1}$. Thus $T_{1} X=0$ and the result holds.

\section{Semi-Slant Submanifolds}

Definition 5.1. $M$ is called a semi-slant submanifold of $\widetilde{M}$ if there exist two orthogonal distributions $D_{1}$ and $D_{2}$ on $M$ such that:

(a) $T M$ admits the orthogonal direct decomposition $T M=D_{1} \oplus D_{2}$.

(b) The distribution $D_{1}$ is invariant distribution, i.e., $F\left(D_{1}\right)=D_{1}$.

(c) The distribution $D_{2}$ is slant with angle $\theta \neq 0$.

In this case, we call $\theta$ the slant angle of submanifold $M$. By virtue of Lemma 4.1, we can see that the invariant distribution of a semi-slant submanifold is slant with zero angle. Thus it is obvious that semi-slant submanifolds are particular cases of bi-slant submanifolds. Furthermore, it is clear that if $\theta=\pi / 2$, then a semi-slant submanifold is a semi-invariant submanifold. On the other hand, if we denote the dimension of $D_{i}$ by $d_{i}$, for $i=1,2$, then we have the following cases:

(a) If $d_{2}=0$, then $M$ is an invariant submanifold.

(b) If $d_{1}=0$ and $\theta=\pi / 2$, then $M$ is an anti-invariant submanifold.

(c) If $d_{1}=0$ and $\theta \neq \pi / 2$, then $M$ is a proper slant submanifold with slant angle $\theta$.

(d) If $d_{1} d_{2} \neq 0$ and $\theta \neq \pi / 2$, then $M$ is a proper semi-slant submanifold.

Given a semi-slant submanifold $M$, we denote by $P_{i}$ the projection on the distribution $D_{i}$ for $i=1,2$. We also put $T_{i}=P_{i} T$. Hence we obtain

$$
F X=F P_{1} X+T P_{2} X+C P_{2} X
$$

for any $X \in T M$. By a direct calculation, we can prove that for any $X \in T M$,

$$
\begin{gathered}
F P_{1} X=T P_{1} X, \quad C P_{1} X=0, \\
T P_{2} X \in D_{2} .
\end{gathered}
$$

In particular, (5.1) and (5.2) imply for any $X \in T M$,

$$
T X=F P_{1} X+T P_{2} X=T P_{1} X+T P_{2} X .
$$

Then from (5.2) and (5.3) we obtain

$$
g\left(T X, T P_{2} Y\right)=\cos ^{2} \theta g\left(X, P_{2} Y\right), \quad g\left(C X, C P_{2} Y\right)=\sin ^{2} \theta g\left(X, P_{2} Y\right)
$$


for any $X, Y \in T M$.

We are going to characterize semi-slant submanifolds by the following theorem.

Theorem 5.1. Let $M$ be a submanifold of a locally product manifold $\widetilde{M}$. Then $M$ is semi-slant if and only if there exists a constant $\lambda \in[0,1)$ such that $\mathcal{D}=\left\{X \in T X \mid T^{2} X=\lambda X\right\}$ is a distribution. Furthermore, in this case, $\lambda=\cos ^{2} \theta$, where $\theta$ denotes the slant angle of $M$.

Proof. Let $M$ be semi-slant and $T M=D_{1} \oplus D_{2}$, where $D_{1}$ is invariant and $D_{2}$ is slant. We put $\lambda=\cos ^{2} \theta$, where $\theta$ denotes the slant angle of $M$. For any $X \in \mathcal{D}$, if $X \in D_{1}$, then $X=F^{2} X=T^{2} X=\lambda X$, which means that $\lambda=1$, but this contradicts that $\lambda \in[0,1)$. So $X \notin D_{1}$ and $\mathcal{D} \subseteq D_{2}$. On the other hand, since $D_{2}$ is a slant distribution, it follows from Theorem 4.1 and Lemma 4.1 that $T^{2} X=\left(P_{2} T\right)^{2} X=\lambda X$, which means that $D_{2} \subseteq \mathcal{D}$. Thus $\mathcal{D}=D_{2}$ is a distribution.

Conversely, we can consider the orthogonal direct decomposition $T M=$ $\mathcal{D} \oplus \mathcal{D}^{\perp}$. It is obvious that $T \mathcal{D} \subseteq \mathcal{D}$, from which we have $g(F X, Y)=$ $g(X, F Y)=g(X, T Y)=0$ for any $X \in \mathcal{D}^{\perp}$ and $Y \in \mathcal{D}$. Hence $\mathcal{D}^{\perp}$ is an invariant distribution. Finally, Lemma 4.1 and Theorem 4.1 imply that $\mathcal{D}$ is a slant distribution, with slant angle $\theta$ satisfying $\lambda=\cos ^{2} \theta$.

Remark. The result above is also valid for almost product Riemannian manifolds, since they do not deal with the Levi-Civita connection and from now on, we are going to deal with semi-slant submanifolds of a locally product manifold. Our goal is to study the integrability.

At first, we will prove the following lemma.

Lemma 5.1. Let $M$ be a semi-slant submanifold of a locally product manifold $\widetilde{M}$. Then for any $X, Y \in T M$, we have:

$$
\begin{gathered}
P_{1}\left(\nabla_{X} F P_{1} Y\right)+P_{1}\left(\nabla_{X} T P_{2} Y\right)=F P_{1}\left(\nabla_{X} Y\right)+P_{1} A_{C P_{2} Y} X \\
P_{2}\left(\nabla_{X} F P_{1} Y\right)+P_{2}\left(\nabla_{X} T P_{2} Y\right)=F P_{2}\left(\nabla_{X} Y\right)+P_{2} A_{C P_{2} Y} X+t \sigma(X, Y), \\
\sigma\left(F P_{1} Y, X\right)+\sigma\left(T P_{2} Y, X\right)+\nabla_{X}^{\perp} C P_{2} Y=C P_{2} \nabla_{X} Y+n \sigma(X, Y) .
\end{gathered}
$$

Proof. Since $\bar{\nabla}_{X} F Y=F \bar{\nabla}_{X} Y$ for any $X, Y \in T M$, by using Gauss-Weigarten formulas we obtain:

$$
\begin{array}{rl}
\nabla_{X} & F P_{1} Y+\sigma\left(F P_{1} Y, X\right)+\nabla_{X} T P_{2} Y+\sigma\left(T P_{2} Y, X\right)-A_{C P_{2} Y} X+\nabla_{X}^{\perp} C P_{2} Y \\
= & P_{1}\left(\nabla_{X} F P_{1} Y\right)+P_{2}\left(\nabla_{X} F P_{1} Y\right)+P_{1}\left(\nabla_{X} T P_{2} Y\right)+P_{2}\left(\nabla_{X} T P_{2} Y\right)-P_{1} A_{C P_{2} Y} X \\
& -P_{2} A_{C P_{2} Y} X+\sigma\left(F P_{1} Y, X\right)+\sigma\left(T P_{2} Y, X\right)+\nabla_{X}^{\perp} C P_{2} Y \\
= & F P_{1} \nabla_{X} Y+T P_{2} \nabla_{X} Y+C P_{2} \nabla_{X} Y+t \sigma(X, Y)+n \sigma(X, Y) .
\end{array}
$$

By comparing the components of $D_{1}, D_{2}$ and $T^{\perp} M$, respectively, we can obtain the above results.

Proposition 5.1. Let $M$ be a semi-slant submanifold of a locally product manifold $\widetilde{M}$. Then we have: 
(a) The distribution $D_{1}$ is integrable if and only if

$$
\sigma(X, F Y)=\sigma(F X, Y)
$$

for any $X, Y \in D_{1}$.

(b) The distribution $D_{2}$ is integrable if and only if

$$
P_{1}\left(\nabla_{X} T Y-\nabla_{Y} T X\right)=P_{1}\left(A_{C Y} X-A_{C X} Y\right)
$$

for any $X, Y \in D_{2}$.

Proof. Let $D_{1}$ be integrable; by using (5.6) we see that

$$
\sigma(X, F Y)-\sigma(F X, Y)=C P_{2}[X, Y]
$$

for any $X, Y \in D_{1}$. Hence if $D_{1}$ is integrable, then (5.7) holds directly from (5.9). Conversely, let $X, Y \in D_{1}$, by using (5.7) and (5.9) it follows that $C P_{2}[X, Y]=$ 0 . So we can easily deduce that $P_{2}[X, Y]$ must vanish, since $D_{2}$ is a slant distribution with nonzero slant angle. Therefore, $[X, Y] \in D_{1}$ and statement (a) holds. As to statement (b), we first compute

$$
F P_{1}[X, Y]=P_{1}\left(\nabla_{X} T Y-\nabla_{Y} T X\right)-P_{1}\left(A_{C Y} X-A_{C X} Y\right)
$$

for any $X, Y \in D_{2}$, by virtue of (5.4). Hence (5.8) holds if and only if $F P_{1}[X, Y]=0$, i.e., $P_{1}[X, Y]=0$, i.e., $D_{2}$ is integrable.

We can also obtain from the above that when $M$ is a semi-invariant submanifold, (b) becomes $P_{1}\left(A_{F Y} X-A_{F X} Y\right)=0$ for any $X, Y \in D_{2}$, which is consistent to the results in $[5]$.

Lemma 5.2. A semi-slant submanifold $M$ of a locally product manifold $\widetilde{M}$ is $D_{i}-D_{j}, i \neq j$, mixed totally geodesic if and only if $A_{N} X \in D_{i}$ for any $X \in D_{i}$, $N \in T^{\perp} M, i=1,2$.

Proof. If $M$ is $D_{1}-D_{2}$ mixed totally geodesic, then for any $X \in D_{1}, Y \in D_{2}$, $N \in T^{\perp} M$,

$$
g\left(A_{N} X, Y\right)=g(\sigma(X, Y), N)=0,
$$

which implies that $A_{N} X \in D_{1}$. Conversely, suppose $A_{N} X \in D_{1}$ for any $X \in D_{1}$, $N \in T^{\perp} M$ and let $\left\{N_{1}, \cdots, N_{m-n}\right\}$ be a local orthogonal basis of $T^{\perp} M$, where $n=\operatorname{dim} M, m=\operatorname{dim} \widetilde{M}$; we have

$$
0=g\left(A_{N_{\alpha}} X, Y\right)=g\left(\sigma(X, Y), N_{\alpha}\right),
$$

$\alpha=1, \ldots, m-n$, for any $X \in D_{1}, Y \in D_{2}$. So $\sigma(X, Y)=0$ and $M$ is $D_{1}-D_{2}$ mixed totally geodesic.

In the same way we can also prove that $M$ is $D_{2}-D_{1}$ mixed totally geodesic if and only if $A_{N} X \in D_{2}$ for any $X \in D_{2}, N \in T^{\perp} M$.

Proposition 5.2. Any invariant submanifold $M$ of a locally product manifold $\widetilde{M}$ is a locally product manifold. 
Proof. Suppose $M$ is an invariant submanifold of a locally product manifold $\widetilde{M}$; then $C \equiv 0$ and we can obtain $\left(\nabla_{X} F\right) Y=t \sigma(X, Y)$, by using (2.7), for any $X, Y \in T M$. On the other hand,

$$
g(Z, t \sigma(X, Y))=g(Z, F \sigma(X, Y))=g(F Z, \sigma(X, Y))=0 .
$$

So we have $t \sigma(X, Y)=0$, which implies $\left(\nabla_{X} F\right) Y=0$ for any $X, Y \in T M$. This is equivalent to saying that $M$ is a locally product manifold.

Proposition 5.3. Let $M$ be a semi-slant submanifold of a locally product manifold $\widetilde{M}$. If $\nabla C \equiv 0$, then $M$ is $D_{1}-D_{2}$ mixed totally geodesic. Furthermore, if $X, Y \in D_{2}$, then either $\sigma(X, Y)=0$ or $\sigma(X, Y)$ is an eigenvector of $n^{2}$ with eigenvalue $\cos ^{2} \theta$. If $X, Y \in D_{1}$, then either $\sigma(X, Y)=0$ or $\sigma(X, Y)$ is an eigenvector of $n^{2}$ with eigenvalue 1 .

Proof. If $\left(\nabla_{X} C\right) Y \equiv 0$ for any $X, Y \in T M$, then from (2.11) we have $f \sigma(X, Y)=\sigma(X, T Y)$. In particular, if $Y \in D_{2}$, then by Lemma 4.1

$$
\begin{aligned}
f^{2} \sigma(X, Y) & =f \sigma(X, T Y)=f \sigma\left(X, T_{2} Y\right)=\sigma\left(X, T T_{2} Y\right) \\
& =\sigma\left(X, T_{2}^{2} Y\right)=\cos ^{2} \theta \sigma(X, Y),
\end{aligned}
$$

where $\theta$ is the slant angle of $D_{2}$. Furthermore, if $X \in D_{1}$, then by Lemma 4.1

$$
\begin{aligned}
f^{2} \sigma(X, Y) & =f^{2} \sigma(Y, X)=f \sigma(Y, T X)=\sigma\left(Y, T^{2} X\right)=\sigma\left(Y, F^{2} X\right) \\
& =\sigma(Y, X)=\sigma(X, Y) .
\end{aligned}
$$

Since $\theta \neq 0$, we have $\sigma(X, Y)=0$ by virtue of (5.13). Hence $M$ is $D_{1}-D_{2}$ mixed totally geodesic. If $X, Y \in D_{i}, i=1,2$, then from (5.13) we can obtain the result easily.

Proposition 5.4. Let $M$ be a mixed totally geodesic semi-slant submanifold of a locally product manifold $\widetilde{M}$. If the distribution $D_{1}$ is integrable, then $F A_{N} X=A_{N} F X$ for any $X \in D_{1}, N \in T^{\perp} M$.

Proof. From (2.3) and (5.7) we have

$$
g\left(F A_{N} X, Y\right)=g\left(A_{N} X, F Y\right)=g(\sigma(X, F Y), N)=g(\sigma(F X, Y), N)
$$

for any $X, Y \in D_{1}$ and $N \in T^{\perp} M$, thus we get $F A_{N} X=A_{N} F X$ by virtue of Lemma 5.2.

\section{ACKNOWLEDGEMENTS}

The authors would like to thank the referee for his suggestions and comments on this paper.

\section{REFERENCES}

1. S. Tachibana, Some theorems on locally Riemannian spaces. Tôhoku Math. J. (2) 12(1960), 281-292.

2. T. Adati, Submanifolds of an almost product Riemannian manifold. Kodai Math. J. 4(1981), No. 2, 327-343. 
3. A. Bejancu, Semi-invariant submanifolds of locally product Riemannian manifolds. $A n$. Univ. Timişoara Ser. Sttiinţ. Mat. 22(1984), No. 1-2, 3-11.

4. A. Bejancu, CR submanifolds of a Kaehler manifold. I. Proc. Amer. Math. Soc. 69(1978), No. 1, 135-142.

5. X. LiU and F.-M. ShaO, Skew semi-invariant submanifolds of a locally product manifold. Portugal. Math. 56(1999), No. 3, 319-327.

6. B. Y. Chen, Slant immersions. Bull. Austral. Math. Soc. 41(1990), No. 1, 135-147.

7. N. Papaghiuc, Semi-slant submanifolds of a Kaehlerian manifold. An. Ştiinţ. Univ. Al. I. Cuza Iaşi Secţ. I a Mat. 40(1994), No. 1, 55-61.

(Received 30.06.2004)

Authors' addresses:

Hongxia Li

Department of Applied Mathematics

Dalian University of Technology, Dalian 116024

P.R. China

Ximin Liu

Department of Applied Mathematics

Dalian University of Technology, Dalian 116024

P.R. China

E-mail: xmliu@dlut.edu.cn

Current address:

Graduate School of Mathematics

The University of Tokyo, 3-8-1, Komaba

Meguro-ku, Tokyo 153-8914

Japan

E-mail: xmliu@ms.u-tokyo.ac.jp 